\title{
Genetic analysis of larval competition in Drosophila melanogaster
}

\author{
Joachim Rodrigues de Miranda and \\ Paul Eggleston
}

Department of Genetics, University of Liverpool, P.O. Box 147, Liverpool L69 3BX.

\begin{abstract}
A chromosome assay was employed to determine the genetic basis of the competitive ability of the larvae of Drosophila melanogaster when competing for a controlled amount of food. The effects of increasing stress due to intra- and inter-genotypic competition were analysed using a yield-density regression analysis producing estimates of the absolute performance at a standard reference density ( $e$-values), the magnitude of intra-genotypic competition and the intergenotypic competitive effect of one genotype on another. A distinction was made between the Sensitivity and the Pressure components of inter-genotypic competition. The probability of survival and mean adult weight were used as measures of competitive success. The genetic analysis revealed high levels of heterosis for the $e$-values and intergenotypic pressure, with correspondingly high inter-chromosomal interactions. Inter-genotypic sensitivity was less consistently heterotic and less epistatic. All dominance was directed towards a competitively superior genotype and both major autosomes were involved in the determination of competitive ability with the greater effect residing on the third chromosome. There was evidence of early non-competitive larval mortality in one of the two sets of substitution lines investigated and the effect of this on the estimation of the genetic parameters is discussed.
\end{abstract}

\section{INTRODUCTION}

The investigation of genetic differences in competitive ability has all the conventional problems of prising genetic information from phenotypic variation, compounded with the problem of accurately measuring the competitive phenotype. If density is synonymous with competitive stress then the sensitivity of a genotype to increasing intra- or inter-genotypic density is a measure of its susceptibility to competition. These interactions are represented by the slopes of a multiple regression analysis relating yield to density (Suehiro and Ogawa, 1980; Mather and Caligari, 1981; Wright, 1981; Spitters, 1983 and Watkinson, 1984). A competition diallel analysis of these interactions (de Miranda and Eggleston, 1987) yields estimates of the effects of intra-genotypic competition (leading diagonal), the Sensitivity of a genotype as indicator competitor to inter-genotypic competition and the Pressure exerted by the genotype as associate competitor on other genotypes during inter-genotypic competition. These quantities differ from the aggression ( $a$ ) and response $(r)$ parameters pre- viously defined by Mather and Caligari (1983) in that their estimation does not involve the intragenotypic competitive values. Considered together, the $e$-value, intra-genotypic effect, intergenotypic pressure and inter-genotypic sensitivity are able completely to describe the competitive profile of a genotype.

The use of density series means that only the mean competitive ability of the genotype is estimated, rather than the performance of each individual, thereby restricting the scope for genetic analysis. A powerful genetic analysis which can be used in such cases is the chromosome assay and examples of various types of chromosome assay used in the analysis of competitive ability are given by Vetukhiv (1953) and Brncic (1954) for coadapted gene complexes in natural populations, Mather and Cooke-(1962) for yields in 50/50 mixtures at two temperatures, Keasey and Kojima (1967) for mean adult weight in inter-related sets of substitution lines and Burnet et al. (1977) for larval feeding rate and its implications in larval competition. Unfortunately, few of these studies exercised the degree of density control necessary for accurate 
determination of the competitive relationships. Here we report the results of two chromosome substitution line analyses of competitive ability encompassing four different second and four different third chromosomes.

\section{EXPERIMENTAL DETAILS}

Full experimental details, including data collection, transformation, regression analysis and diallel analysis can be found in de Miranda and Eggleston (1987). Briefly, competition took place in glass vials containing $5 \mathrm{ml} 2$ per cent bacto-agar and $55 \mathrm{mg}$ yeast (YSC-2, Sigma) dispensed as a solution. The vials were seeded with even aged eggs of the various genotypes, using $(30,0),(60,0)$, $(90,0)$ and $(120,0)$ eggs per vial for the monoculture density series and $(30,90),(60,60)$ and $(90,30)$ eggs of either genotype per vial for each duoculture density series. Two duoculture density series were raised for each wild type genotype, one with $y^{2} \mathrm{~T} 19$ and one with $y^{2} \mathrm{~T} 25$. The yellow body colour marker $\left(y^{2}\right)$ carried by the two tester genotypes makes it possible to distinguish the competitors in duoculture (Eggleston, 1987). The susceptibility of the wild type genotype to increasing intra-genotypic competition was represented by the slope of the regression of performance onto monoculture density. For the duoculture series a similar slope can be calculated, considering the wild type genotype as the indicator, which meets the monoculture slope at density $(120,0)$. If the complementing numbers of yellow associates have no effect on the performance of the wild type indicator then the mono- and duoculture slopes will coincide. The difference between the slopes can therefore be attributed to the inter-genotypic pressure of the yellow associate on the wild type indicator. Alternatively, the difference can be interpreted as the inter-genotypic sensitivity of the wild type indicator to the pressure it faces from the yellow associate. A similar analysis of competitive performance, taking the yellow genotype as the indicator, will estimate the inter-genotypic pressure of the wild type associate and the intergenotypic sensitivity of the yellow indicator. For each wild type genotype two duoculture series were raised with different yellow tester strains and hence the means of the relevant parameters were used in the genetic analyses. The $e$-values, representing the absolute performance of each genotype at the reference density of $(120,0)$ and the monoculture slopes, representing the effects of intra-genotypic competiton, were also investigated. Two competi- tive characters were scored, namely the proportion of seeded eggs surviving to adulthood, transformed to angles $\left(p_{a}\right)$ and the mean weight of surviving adults $(\bar{w})$, transformed to $(1 / \bar{w})$ for regression purposes (de Miranda and Eggleston, 1987). The genetic analysis of $e$-values for mean adult weight is presented in terms of $(\bar{w})$ rather than $(1 / \bar{w})$ for ease of interpretation.

The chromosome substitution lines were constructed using standard techniques which involved the multiply inverted balancer strain $c 23_{a}$. This has the constitution; In $(2 L+2 R) C y$; $C y \mathrm{cn}^{2} / \mathrm{In}$ (2LR) Pmal $\mathrm{Ps}^{43 k} l_{t} b w^{v i}$ and In (3LR) $D c x F ; D / S b$ (see Lindsley and Grell, 1967 for further description). These inversions minimise recombination such that wild type chromosomes can be transferred largely intact. The inbred lines T5 and T27 (set T5-T27) and T19 and T25 (set T19-T25) supplied the wild type chromosomes (see Linney et al., 1971 for the origin of this material). The substitution lines were sib-mated for five generations prior to their use in these experiments. For each set of substitution lines a subset of four lines, representing variation for the second and third chromosomes was investigated. Due to the size of the experiment variation for the $\mathrm{X}$ and fourth chromosomes was not investigated in these experiments. The small fourth chromosome, however, is generally accepted as having little genetic activity and the $\mathrm{X}$ chromosomes from the parental strains were distributed randomly among the substitution lines and would not be expected to interfere with the interpretation for chromosomes 2 and 3. For each subset a half diallel of ten crosses was raised in duplicate on each of two occasions with the reciprocal versions of each cross allocated at random to either occasion (see fig. 1). Differences between the occasions were generally not significant but some of the reciprocal differences for the T19-T25 subset were significant. This non reciprocity was due to a larval mortality factor linked to the maternal T19 third chromosome and was found for all crosses producing heterozygous third chromosomes. This effect was not found, however, when either 19D or the original T19 inbred line was involved. Whether reciprocal differences are present or not, each genotype is most accurately represented by the mean of its reciprocals. We chose to treat the data as a half diallel with four replicates. Each genotype was analysed independently with the differences between the four replicates providing the basis for all error variances associated with the competitive parameters of that genotype. Naturally, the presence of reciprocal differences for the off- 


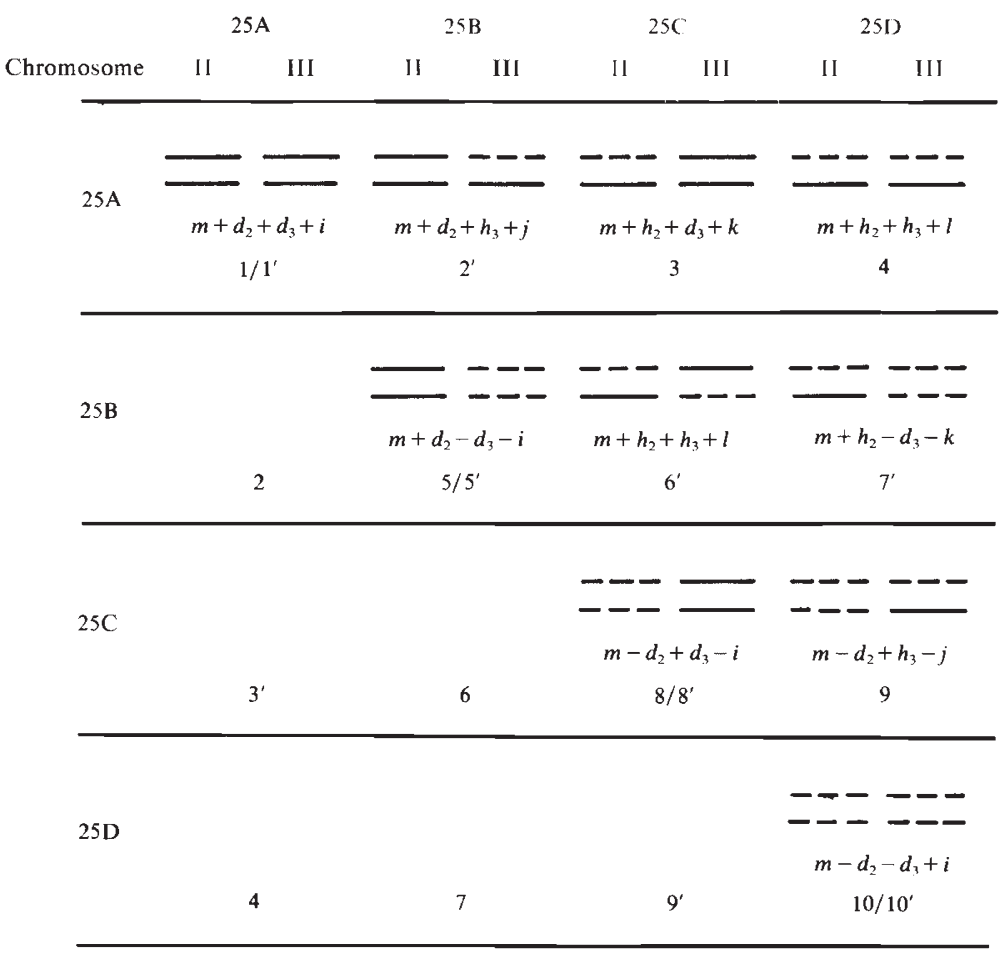

Figure 1 Schematic representation of the genetic analysis of the half diallel. Solid lines represent chromosomes derived from T19 and broken lines those from T25. The genotypes are identified by numbers 1-10 (occasion 1) and $1^{\prime}-10^{\prime}$ (occasion 2) and the genetic parameters are defined in Table 1 and in the text.

diagonal genotypes in the half diallel will inflate their particular error variances. Apart from overestimating the average error variance and thereby placing extra emphasis on significant results, this variation in the error variances can be usefully employed in a variance weighted analysis of the half diallel. Each of the ten genotypes was defined according to its genetic constitution as shown in fig. 1 , where $m$ is the overall mean, $d_{2}$ and $d_{3}$ and $h_{2}$ and $h_{3}$ are the additive and dominance deviations for the second and third chromosomes respectively and $i, j, k$ and $l$ are the additiveadditive, additive-dominance, dominance-additive and dominance-dominance interactions respectively. The nine genetic parameters were estimated from the ten genotypes using a variance weighted least squares procedure, which biases against items with large reciprocal differences. The remaining degree of freedom was used to estimate the residual variation which estimates interchromosomal interactions not specified in fig. 1 . In a variance weighted analysis items with large error variances, which contribute relatively little to the estimation of the genetic parameters, are more likely to deviate from their expected values. Hence, the following modified $\chi^{2}$ formula was used to account for this bias;

$$
\chi_{(1)}^{2}=\sum_{i=1}^{i=10}\left(O_{i}-E_{i}\right)^{2} / s_{i}^{2}
$$

where $s_{i}^{2}$ substitutes for the more conventional $s^{2}$, $O_{i}, E_{i}$ and $s_{i}^{2}$ refer to the observed, expected and error variance estimates of each item. The error variances associated with the genetic parameters are themselves weighted estimates, obtained essentially as the harmonic mean of the component error variances (Snedecor and Cochran, 1980) and are found on the leading diagonal of the inverted coefficient matrix. The T19 and T5 derived chromosomes were regarded a priori as the increasing component throughout (see fig. 1). Thus, a positive deviation for the additive differences indicates a larger value for the T19 or T5 chromosome. Finally, genotypes $4 / 4^{\prime}$ and $6 / 6^{\prime}$ in the half diallel (fig. 1 ) are genetically identical but are derived from different parents and their similarity is evidence for the fidelity with which the chromosomes are transferred into different genetic backgrounds (Caligari and Mather, 1975) as is the similarity of the reconstituted genotypes 27D and 25D to the original inbreds T27 and T25. The fortuitous 
Table 1 Estimates of the genetic parameters and error variances derived from the competitive interactions among the substitution line subsets T19-T25 (a) and T5-T27 (b) for larval survival $\left(p_{a}\right)$ and mean adult weight $(1 / \bar{w} ; \bar{w})$

(a) $\mathrm{T} 19-\mathrm{T} 25$

\begin{tabular}{|c|c|c|c|c|c|c|c|c|}
\hline & & & $p_{a}$ & & $\bar{w}$ & & $1 / \bar{w}$ & \\
\hline & $e$-value & $C_{X X}$ & $C_{Y X}$ & $C_{X Y}$ & $e$-value & $C_{X X}$ & $C_{Y X}$ & $C_{X Y}$ \\
\hline$m$ & $32.7575 * * *$ & $15 \cdot 8193 * * *$ & $7 \cdot 9969 * * *$ & $13 \cdot 5982 * * *$ & $40 \cdot 4250 * * *$ & $17 \cdot 564 * * *$ & $16 \cdot 8548 * * *$ & $17 \cdot 9069 * * *$ \\
\hline$d_{2}$ & $4.06 \delta 0 * * *$ & $-10 \cdot 2275^{* * *}$ & -1.1349 & $-3 \cdot 1028$ & $2 \cdot 1160$ & $-3 \cdot 6600^{* * *}$ & $-2 \cdot 8958$ & 0.7351 \\
\hline $\operatorname{var}\left(d_{2}\right)$ & 0.932 & $3 \cdot 591$ & $3 \cdot 386$ & 1.690 & 0.876 & 0.889 & 1.859 & 0.418 \\
\hline$d_{3}$ & $-4.9631^{* * *}$ & $-15 \cdot 1546^{* * *}$ & $-7 \cdot 0248 * * *$ & $-6 \cdot 7035^{* * *}$ & $15 \cdot 0611^{* * *}$ & $-9 \cdot 3894^{* * *}$ & $-10 \cdot 2131^{* * *}$ & $-1 \cdot 0254$ \\
\hline $\operatorname{var}\left(h_{2}\right)$ & 3.059 & $11 \cdot 791$ & $10 \cdot 159$ & $5 \cdot 549$ & $3 \cdot 551$ & $3 \cdot 225$ & $5 \cdot 579$ & $1 \cdot 518$ \\
\hline$h_{3}$ & $9 \cdot 4625^{* *}$ & 6.4298 & $13 \cdot 1434^{* * *}$ & $-1 \cdot 5853$ & $-11 \cdot 1666^{* * *}$ & $6 \cdot 6312$ & $12 \cdot 1025^{* * *}$ & $-3 \cdot 1202$ \\
\hline $\operatorname{var}\left(h_{3}\right)$ & $10 \cdot 824$ & $60 \cdot 290$ & $10 \cdot 159$ & $25 \cdot 323$ & 4.476 & $7 \cdot 530$ & $5 \cdot 579$ & $3 \cdot 544$ \\
\hline$i$ & $-4 \cdot 8968 * * *$ & $9.4332^{* * *}$ & 0.7747 & $6.4066^{* * *}$ & $-3.0877^{* *}$ & $2 \cdot 8096^{* *}$ & $1 \cdot 1613$ & $1 \cdot 7382$ \\
\hline $\operatorname{var}(i)$ & 0.932 & $3 \cdot 591$ & $3 \cdot 386$ & $1 \cdot 690$ & 0.876 & 0.889 & 1.859 & 0.418 \\
\hline$j$ & $-3 \cdot 8878$ & $1 \cdot 7472$ & $-8 \cdot 3153$ & 0.8497 & -0.4010 & 0.4079 & $-2 \cdot 2153$ & -2.0611 \\
\hline $\operatorname{var}(l)$ & $15 \cdot 836$ & $79 \cdot 527$ & $23 \cdot 703$ & $34 \cdot 414$ & 8.969 & $14 \cdot 747$ & $13 \cdot 018$ & 6.940 \\
\hline$\chi_{(1)}^{2}$ & $4 \cdot 48^{*}$ & 0.00 & $30.07^{* * *}$ & $0 \cdot 11$ & $1 \cdot 89$ & $2 \cdot 71$ & $4.92 *$ & $4 \cdot 11^{*}$ \\
\hline
\end{tabular}

(b) T5-T27

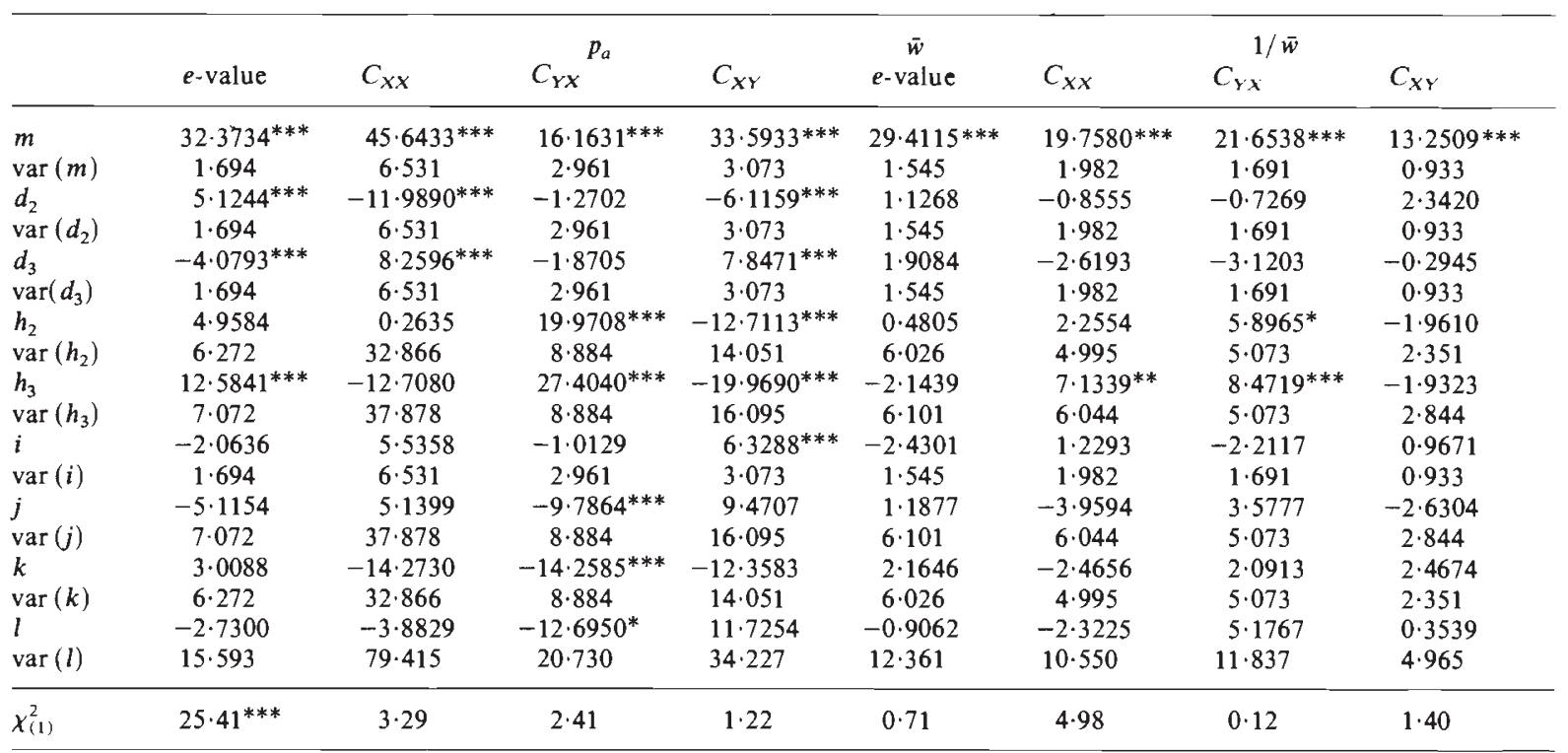

Referring to the wild type genotypes as $X$ and the yellow marked tester genotypes as $Y$, genetic parameters are given for the $e$-values, intra-genotypic competition among wild type individuals $\left(C_{X X}\right)$, inter-genotypic competitive pressure of wild type associate on yellow indicator $\left(C_{Y X}\right)$ and inter-genotypic competitive sensitivity of wild type indicator to yellow associate ( $\left.C_{X Y}\right)$. The parameters $d_{2}$ and $d_{3}$ refer to the additive differences on chromosomes 2 and 3 respectively, $h_{2}$ and $h_{3}$ similarly refer to the dominance deviations for each chromosome. $i, j, k$ and $l$ refer to the additive-additive, additive-dominance, dominance-additive and dominance-dominance inter-chromosomal interaction items. To facilitate presentation the estimates and variances for $C_{X X}, C_{Y X}$ and $C_{X Y}$ in $p_{a}$ and the $e$-value in $\bar{w}$ were multiplied by $10^{2}$ and $10^{4}$ respectively. Similarly, the estimates and variances for $C_{X X}, C_{Y X}$ and $C_{X Y}$ in $1 / \bar{w}$ were multiplied by $10^{3}$ and $10^{6}$ respectively.

Levels of significance were determined by $t$-test and are given as ${ }^{*}, 0.05>P>0.01 ; * *, 0.01>P>0.001$ and ${ }^{* * *}, P<0.001$. Interchromosomal interactions not specified in fig. 1 are tested as $\chi_{(1)}^{2}$. 
presence of recognisable markers (larval mortality on the T19 third chromosome and $p^{p}$ on the T5 third chromosome) also provides a test of the accuracy of the crossing procedure. The data suggest that the various chromosomes were transferred with the minimum of disruption.

\section{RESULTS}

The results of the genetic analysis are given in table 1(a) and (b) for substitution line sets T19-T25 and T5-T27 respectively and it is evident that all types of genetic variation and interaction are represented among the four competitive parameters. Some caution must be exercised when considering the dominance parameters (principally $h$ and $l$ ) since the inherantly larger error variances tend to reduce their levels of significance in comparison with the additive parameters. This is in itself not surprising since three genotypes (two homozygotes and their $F_{1}$ ) are required to obtain a dominance deviation whilst the additive deviation involves the comparison of only two homozygotes.

A brief discussion of the four competitive parameters may facilitate the interpretation of the data. Characteristics associated with competitive strength are high estimates for inter-genotypic pressure in $p_{a}$ and $1 / \bar{w}$ (representing the success of a genotype in depressing the performance of the yellow indicator genotype) and for the $e$-value in $p_{a}$ (representing a high probability of survival at the highest density). Low estimates for intergenotypic sensitivity in $p_{a}$ and $1 / \bar{w}$ (representing the susceptibility of a genotype to competitive pressure) and for the $e$-value in $\bar{w}$ also indicate competitive strength. Since survival is limited at the highest density, the mean adult weight at this density ( $e$-value for $\bar{w}$ ) is probably closely related to the minimum larval weight required for successful pupation (Bakker, 1961; 1969). Clearly, a low critical weight can only be advantageous. Logic suggests that the estimates for intra-genotypic competition are closely related to the $e$-values. For example, if low density survivial is the same for all genotypes then the value for high density survival ( $e$-value) will determine the slope obtained in monoculture. A similar argument can be made with respect to mean adult weight. In these experiments, with evidence of density independent larval mortality, low density survival was not the same for all genotypes. Consequently, no correlation was found between the $e$-values and intragenotypic competition for survival $\left(r_{18}=0 \cdot 259\right)$. However, highly significant negative correlation was found between the weight related $e$-values and intra-genotype competition $\left(r_{18}=-0.905\right)$. Since both pressure and sensitivity, as defined in this report, reflect the inter-genotypic competitive ability of a genotype we may expect them to be correlated to some extent. Table 2 shows the correlations between the various estimates of inter-genotypic pressure and sensitivity derived from the 20 genotypes in two substitution line analyses. Although these coefficients have the expected sign for both survival $\left(r_{18}=-0.028\right)$ and adult weight $\left(r_{18}=-0.431\right)$, neither is significant. This suggests that inter-genotypic pressure and sensitivity are effectively independent as found for the related parameters aggression $(a)$ and response $(r)$ previously described by Mather and Caligari (1983); Eggleston (1985) and Hemmat and Eggleston (1988). These investigations imply that intergenotypic pressure and sensitivity (and therefore aggression and response) may have a different biological basis and this hypothesis has been investigated further by de Miranda and Eggleston $(1988 a, b)$.

Inspection of table 1 reveals that competitive ability as a whole is strongly influenced by dominance and heterosis, as well as additive effects. Inter-genotypic competitive pressure consistently displays high levels of positive heterosis whereas the $e$-values have a less pronounced tendency towards heterosis, positive for $p_{a}$ and negative for $\bar{w}$. Inter-genotypic sensitivity has more intermediate levels of negative dominance which rarely extends to heterosis. Intra-genotypic competition generally deviates in the opposite direction to the $e$-values which is as expected from the arguments in the previous section. Both chromosomes are involved in the determination of competitive ability with the greater effect residing on the third chromosome and all dominance and heterosis is directed toward a competitively superior genotype.

Table 2 Correlation coefficients for 18 degrees of freedom between the estimates of inter-genotypic competitive pressure $\left(C_{Y X}\right.$ as defined in table 1$)$ and inter-genotypic competitive sensitivity $\left(C_{X Y}\right.$ as defined in table 1$)$ for two competitive characters, namely larval survival $\left(p_{a}\right)$ and mean weight of surviving adults $(1 / \bar{w})$

\begin{tabular}{cccccc}
\hline & & \multicolumn{2}{c}{$p_{a}$} & \multicolumn{2}{c}{$1 / \bar{w}$} \\
\hline$p_{a}$ & & $C_{Y X}$ & $C_{X Y}$ & $C_{Y X}$ & $C_{X Y}$ \\
& $C_{Y X}$ & 1.00 & -0.028 & $0.813^{* * *}$ & $-0.621^{* *}$ \\
& $C_{X Y}$ & & 1.00 & 0.001 & -0.126 \\
& $C_{Y X}$ & & & 1.00 & -0.431 \\
& $C_{X Y}$ & & & & 1.00 \\
\hline
\end{tabular}

Tests of significance are given as in table 1 . See text for details. 
There is some evidence for gene dispersion among the original inbred lines, as indicated by the contrasting additive effects of the different chromosomes. This may partly explain the observed levels of heterosis if dominance is uni-directional at the majority of loci. Inspection of the interaction items in table 1 reveals a further trend. More often than not the dominance-dominance interaction $(l)$ is opposite in sign to the direction of the dominance itself and this is especially evident when dominance is large. For example, the dominance-dominance interaction for inter-genotypic pressure is negative while dominance is positive. The $e$-values in $p_{a}$ show a similar trend while for inter-genotypic sensitivity the interaction was positive where dominance was negative. Few of these interaction items are statistically significant, however, and any interpretation must be undertaken with caution. If this trend proves to be genuine then the implication that dominance on either chromosome is enough to approach the optimum phenotype, for a range of characters, may be of interest.

A highly diagnostic feature of the T19 inbred line is the presence of non-competitive larval mortality which removes more than 50 per cent of the larvae at some stage during larval development. If this happened late in the larval phase, after food exhaustion, the competitive relations would be little affected and only the $e$-value in $p_{a}$ would be reduced. Early larval death, on the other hand, would have a significant impact on the competitive parameters, especially in $p_{a}$. The resource would no longer be extremely limiting and maximum survival throughout the density series would be a distinct possibility for all genotypes. The implication is that no additive or dominance differences would be found for any of the competitive parameters in $p_{a}$. The possible effects of larval mortality on $1 / \bar{w}$ are less pronounced since this concerns events which take place after survival has been assured. Even so, the $e$-value in $\bar{w}$ would no longer be very closely related to the critical weight, especially if survival was maximised throught the density series, and would consequently be slightly larger than expected. To test whether larval mortality occurred early or late in the larval phase all possible homozygous and heterozygous genotypes in the T19-T25 set of substitution lines carrying a homozygous T19 third chromosome (and therefore displaying larval mortality) were raised in a single experiment. No variation of note was obtained for any of the competitive parameters in $p_{a}$ and only slightly more for the parameters in $1 / \bar{w}$. There was effectively no density response for all genotypes in $p_{a}$ and the mean weight of the emerging adults at the standard reference density (genetic parameter $m$; $e$-value in $\bar{w}$ ) was well above that found for the experiments reported here. These results suggest early, rather than late, larval mortality and indicate that part of the genetic effects associated with chromosome 3 in the T19-T25 subset will be due to non-competitive larval mortality rather than competitive differences. How large an effect larval mortality might have on the estimation of genetic parameters is difficult to assess. It is unlikely that the adult weight related genetic parameters will be much affected, as indicated above. We could also expect the inter-genotypic pressure and sensitivity parameters to be less affected since their estimation involves the yellow tester genotypes as well as the wild type substitution lines.

\section{DISCUSSION}

The high levels of non-additive genetic variation (dominance, heterosis and epistasis) found for the competitive parameters investigated in these experiments have often been predicted for fitness related characteris (Robertson, 1955; Mather, 1983). All dominance and heterosis was directed towards competitive superiority and this may have a variety of causes. The alleles at those genes influencing competitive ability may have been dispersed throughout the population at the time that the inbred lines were created such that none of the homozygous inbreds possessed the optimum array of alleles. The $F_{1}$ hybrid of any two inbred lines would therefore tend to display heterosis if dominance were unidirectional at the majority of loci. The apparent ease with which deleterious recessive alleles can spread through natural and laboratory populations (Temin et al., 1969; Yamazaki and Hirose, 1984) especially after recent population bottle-necks (Chadburn, 1986) may also contribute to the observed levels of heterosis. Another point of interest is the possible influence of non competitive larval mortality on the estimation of the genetic parameters. The reduction in effective density associated with early larval death ensures that all remaining larvae in monoculture (and to a lesser extent in duoculture) can survive, irrespective of genotype. As a consequence, there is no significant variation between the genotypes, especially for the survival related parameters. This implies that the estimated genetic variation is determined by the level of environmental stress, simulated in these experiments by culture density but more accurately 
involving also the environmental quality and the genotypic requirements. Similar observations were made by Robertson (1961) and Sang (1964) with respect to the stress induced by sub-optimal artificial larval diets. A wider implication is that the competitive fitness differences of the genotypes are stress or density dependent. This becomes especially interesting when considering that laboratory populations carry their variation largely in heterozygous form, despite the enormous directional selection pressure for competitive ability implied by the high levels of density related larval mortality. The possible influence of stochastic effects in competition may provide an explanation. Experiments in plant competition suggest that the size and competitive ability of a plant are positively correlated such that small, random differences in seedling size can be magnified exponentially by the competitive process (Salter et al., 1981; Benjamin, 1982; Cannell et al., 1984). Given the extent of variation for larval age in natural populations, this means that the difference between fit and unfit genotypes would be less distinct. Thus, potentially inferior genotypes could use a head start to survive to adulthood and contribute to the following generation whereas competitively superior genotypes, already being assured of survival, would benefit less from such an advantage. Naturally, the effect of such stochastic competitive processes on the genetic constitution of a population would be influenced by the variation in larval age, the level of competitive stress and the relationship between larval age and competitive ability.

Acknowledgements We are grateful for financial support from the SERC (Research Studentship to J. R. de Miranda) and the Lister Institute of Preventive Medicine (Research Fellowship to P. Eggleston).

\section{REFERENCES}

BAKKER, K. 1961. An analysis of factors which determine success in competition for food among larvae of Drosophila melanogaster. Arch. Neerl. Zool., 14, 200-281.

BAKKER, K. 1969. Selection for rate of growth and its influence on competitive ability of larvae of Drosophila melanogaster. Neth. J. Zool., 19, 541-595.

BENJAMIN, L. R. 1982. Some effects of differing times of seedling emergence, population density and seed size on rootsize variation in carrot populations. J. Agric. Sci. Camb., 98, 537-545.

BRNCIC, D. 1954. Heterosis and integration of genotypes in geographic populations of Drosophila pseudo-obscura. Genetics, 39, 77-88.
BURNET, B., SEWELL, D. AND BOS, M. 1977. Genetic analysis of larval feeding behaviour in Drosophila melanogaster. II Growth relations and competition between selected lines. Genet. Res. Camb., 30, 149-161.

CALIGARI, P. D. S. AND MATHER, K. 1975. Genotype-environment interaction. II. Interactions in Drosophila melanogaster. Proc. Roy. Soc. Lond. B., 191, 387-411.

CANNELL, M. G. R., ROTHERY, P. AND FORD, E. D. 1984. Competition within stands of Picea sitchensis and Pinus contorta. Ann. Bot., 53, 349-362.

CHADBURN, R. G. 1986. An investigation into the genetics and ecology of a closed semi-natural population of Drosophila melanogaster. Ph.D Thesis, University of Liverpool.

EGGLESTON, P. 1985. Variation for aggression and response in the competitive interactions of Drosophila melanogaster. Heredity, 54, 43-51.

EGGLESTON, P. 1987. The use of genetic markers in the analysis of competitive interactions in Drosophila melanogaster. Genetica, 72, 181-186.

HEMMAT, M. AND EGGleston, P. 1988. Competitive interactions in Drosophila melanogaster: recurrent selection for aggression and response. Heredity, 60, 129-137.

KEARSEY, M. J. AND KOJIMA, K-I. 1967. The genetic architecture of body weight and egg hatchability in Drosophila melanogaster. Genetics, 56, 23-37.

LINDSLEY, D. I. AND GRELL, E. H. 1967. Genetic variations of Drosophila melanogaster, Carn. Inst. Wash. Publ., 627.

LINNEY, R., BARNES, B. W. AND KEARSEY, M. J. 1971. Variation for metrical characters in Drosophila populations. Heredity, 27, 163-174.

MATHER, K. 1983. Response to selection. In: Ashburner, M., Carson, H. L. and Thompson, J. N. (eds) The Genetics and Biology of Drosophila, vol. 3c, Academic Press. London.

MATHER, K. AND CAligARI, P. D. S. 1981. Competitive interactions in Drosophila melanogaster. II. Measurement of competition. Heredity, 46, 239-254.

MATHER, K. AND CALIGARI, P. D. S. 1983. Pressure and response in competitive interactions. Heredity, 51, 435-454.

MATHER, K. AND COOKE, P. 1962. Differences in competitive ability between genotypes of Drosophila. Heredity, 17, 381407.

de MiRANDA. J. R. AND EGGLESTON, P. 1987. A comparison of substitution and addition designs for the analysis of competitive interactions in Drosophila melanogaster. Her. edity, 58, 279-288.

de MIRANDA, J. R. AND EGGleSton, P. 1988a. Larval competition in Drosophila melanogaster. I. Estimation of larval growth parameters. Heredity, 60, 205-212.

de MIRANDA, J. R. AND EGgleston, P. 1988b. Larval competition in Drosophila melanogaster. II. Comparing biological and competitive parameters. Heredity, 60, 213-219.

ROBERTSON, A. 1955. Selection in animals: synthesis. Cold Spring Harbour Symp. Quant. Biol., 20, 225-299.

ROBERTSON, F. W. 1961. The ecological genetics of growth in Drosophila. 4. The influence of larval nutrition on the manifestation of dominance. Genet. Res. Camb. 2, 346-360.

SALTER, P. J., CURRAH, I. E. AND FELLOWS, J. F. 1981. Studies on some sources of variation in carrot root weight. J. Agric. Sci. Camb., 96, 549-556.

SANG, J. H. 1964. Nutritional requirements of inbred lines and crosses of Drosophila melanogaster. Genet. Res. Camb. 5, 50-67.

SNEDECOR, G. W. AND COCHRAN, W. G. 1980. Statistical Methods. Iowa State University Press. 
SPITTERS, C. J. T. 1983. An alternative approach to the analysis of mixed cropping experiments. I. Estimation of competition effects. Neth. J. Agric. Sci., 31, 1-11.

SUEHIRO, K. AND OGAWA, H. 1980. Competition between two annual herbs, Atriplex gmelini C. A. Mey. and Chenopodium album $L$. in mixed cultures irrigated with sea water of various concentrations. Oecologia, 45, 167-177.

TEMIN, R. G., MEYER, H. U., DAWSON, P. S. AND CROW, J. F. 1969. The influence of epistasis on homozygous viability depression in Drosophila melanogaster. Genetics, 61, 497519.

VETUKHIV, M. 1953, Viability of hybrids between local populations of Drosophila pseudo-obscura. Proc. Natl. Acad. Sci., $39,30-34$.
WATKINSON, A. R. 1984, Yield-density relationships: The influence of resource availability on growth and self thinning in populations of Vulpia fasciculata. Ann. Bot., 53, 967-976.

WRIGHT, A. J. 1981. The analysis of yield-density relationships in binary 'mixtures using inverse polynomials. J. Agric. Sci. Camb., 96, 561-567.

YAMAZAKI, T. AND HIROSE, Y. 1984. Genetic analysis of natural populations of Drosophila melanogaster in Japan. II. The measurement of fitness components in homozygous lines. Genetics, 108, 213-221. 